\title{
377. Neue Aspekte zur histologischen Aufarbeitung experimenteller Darmanastomosen
}

\author{
J. Waninger ${ }^{1}$, I. Shah ${ }^{2}$ und M. Heinz ${ }^{1}$ \\ ${ }^{1}$ Chirurgische Universitätsklinik Freiburg, ${ }^{2}$ Pathologisches Institut, Universität Freiburg, \\ Hugstetter Straße 55, D-7800 Freiburg
}

\begin{abstract}
New Aspects for Histologic Examination of Experimental Anastomoses
Summary. The histologic reaction was examined in suture loops of experimental intestinal anastomoses. A special procedure was developed. Sections were taken from the knot area and from between the knots, as well as longitudinal to the anastomosis. Colonic anastomoses were sutured in male Wistar rats in accordance with eight technical parameters (apposition, number of layers, stitch, distance from the wound edge, distance between knots, type and position of the knot, suture tension, suture material). The suture tension was varied in only three strengths (no tension and medium and strong tensions). The tissue damage due to the suture tension could thus be demonstrated.
\end{abstract}

Key words: Histologic examination - Experimental anastomosis - Suture tension - Technical parameters.

Zusammenfassung. Die Gewebereaktion wurde in der Fadenschlaufe experimenteller Darmanastomosen histologisch untersucht. Hierzu wurde ein Schema entwickelt. Schnitte wurden quer zur Anastomose direkt am Knoten sowie zwischen zwei Knoten und außerdem längs dazu gelegt. Dickdarmanastomosen männlicher Wistar-Ratten wurden unter Berücksichtigung von 8 technischen Parametern (Adaptation, Nahtreihe, Stichführung, Einstichabstand, Knotenabstand, Knotenform und -lage, Nahtspannung, Nahtmaterial) standardisiert genäht. Lediglich die Nahtspannung wurde in 3 Größen verändert (ohne Spannung, mittlere und starke Nahtspannung). Die Quetscheffekte, die durch die Nahtspannung entstanden, konnten auf diese Weise dargestellt werden.

Schlïsselwörter: Histologische Untersuchung - Experimentelle Anastomosen - Nahtspannung - Technische Parameter.

\section{Die NMR-Untersuchung des Körperstammes und der Extremitäten}

\author{
N. Rupp, M. Reiser und E. Stetter \\ Institut für Röntgendiagnostik (Direktor: Prof. Dr. H. Anacker) der Technischen Universität München, \\ Siemens UB Med Erlangen, Ismaninger Straße 22, D-8000 München 80
}

\section{The NMR Examination of the Trunk and the Extremities}

Summary. NMR examination using a Siemens NMR-prototype in 67 patients gave the following results: good demonstration of anatomical structures; complete spatial orientation by tomography in any desired plane; clear distinction between normal and pathologic tissue; delineation of heart and vessels without use of contrast medium; tissue characterisation on the basis of relaxation times; examination using different sequence times are mandatory; no ionizing radiation, no harmful effects known.

Key words: NMR examination - Imaging techniques - Tumor diagnosis.

Zusammenfassung. NMR-Untersuchungen durchgeführt mit einem Siemens NMR-Prototyp an 67 Patienten ergaben: gute Darstellung anatomischer Strukturen, komplette räumliche Orientierung durch Schichtung in jeder beliebigen Ebene, klare Unterscheidung von normalem und pathologischem Gewebe, Herz und Gefäße grenzen sich ohne Kontrastmittel ab, Gewebecharakterisierung auf Grund der Relaxationszeiten, Untersuchungen bei verschiedenen Sequenzzeiten unentbehrlich, keine ionisierenden Strahlen, keine schädigende Wirkung bekannt.

Schliisselwörter: NMR-Untersuchungen - Kernspin-Tomographie - Bildgebende Verfahren - Tumordiagnostik. 